\title{
Target enumeration via integration over planar sensor networks
}

\author{
Yuliy Baryshnikov \\ Mathematical and Algorithmic Sciences \\ Bell Laboratories \\ Murray Hill NJ, USA
}

\author{
Robert Ghrist \\ Department of Mathematics and \\ Coordinated Sciences Laboratory \\ University of Illinois \\ Urbana IL, USA
}

\begin{abstract}
We solve the problem of counting the total number of observable targets (e.g., persons, vehicles, etc.) in a region based on local counts performed by sensors which measure only the number of targets nearby and neither their identities nor any positional information. This theory is robust and accommodates ad hoc sensor networks and mobile robot sensors alike.
\end{abstract}

\section{INTRODUCTION: TARGET COUNTING}

The prospect of small-scale sensor devices comes with the promise of sensor networks which can survey a region with dense coverage [5]. With this promise, however, comes many challenges, including power consumption, heat dissipation, and communication complexity. One strategy for remedying the situation is to focus on minimal sensing, engineering the individual sensors to be as simple as possible to accomplish the task and yet consume a minimum of resources.

We consider how to solve a simple data aggregation problem - counting unidentified targets - with a network of local minimal sensors. Specifically, we show that one can solve enumeration problems with sensors that can count nearby targets but cannot determine target identities, cannot estimate target range or bearing, and cannot record a time when a (moving) target came into view. Because the local sensors we envisage cannot discriminate targets, it is not obvious how to merge redundant counting by neighboring nodes.

It may seem surprising that a redundant array of simplistic sensors can solve the global enumeration problem. More surprising still is the fact that there are very few requirements on the sensors' detection specifications. We do not require that target visibility is purely a function of distance (cf. the typical use of the unit disc assumption in coverage problems). There are no hidden assumptions about convexity of the targets' detection zones, nor that the sensors or targets are uniform: some targets may have more 'impact' than others.

The reason for this combination of extreme robustness and simplicity of the sensor capabilities is the nature of our solution methods. We use a topological invariant - the Euler characteristic - molded into an integration theory.

\section{A. Related work}

There are few similar approaches to problems in target estimation or tracking, the literature on which almost always assumes the ability to identify different targets (along with other high-level functions, including distance estimation, bearing estimation, and sensor localization). For example, the large-scale wireless system implemented in [10] assumes an aggregation phase based on strict spatial separation of targets. Jung and Sukhatme [11] implement a multi-target robotic tracking system where the targets are labeled with colored lights. The survey paper of Guibas [8] pointing to the broader literature on geometric range-searching assumes the ability to aggregate target identities and concerns itself with computational complexity issues. The paper by $\mathrm{Li}$ et al. [13] on multi-target tracking via sensor networks notes that, "target classification is arguably the most challenging signal processing task in the context of sensor networks."

We are aware of two notable exceptions. One significant solution to a target enumeration problem is found in the work of Fang, Zhao, and Guibas [6], which gives a distributed algorithm for target enumeration without any target-identification capabilities on the part of the sensors. Their work assumes that all target supports are round balls in $\mathbb{R}^{2}$; that each sensor reads a $\mathbb{R}$-valued signal proportional to the inverse square of distance-to-target; and that target impacts are additive. Their algorithm counts the number of local maxima in the sensor signal field and therefore gives an accurate count so long as the target supports overlap minimally or not at all. Our work is complementary to this in that the theory we introduce is designed to handle very complex target support overlaps.

The other example of target counting without identification or localization arises in work of Singh et al., who consider a network of sensors which return a value in $\{0,1\}$ depending on target proximity [18]. Their technique involves using timeseries data in the case of moving targets/sensors, since a target count in the stationary case is too difficult, even if all target supports are convex, round, fixed, etc.

We have ignored for the moment many of the important technical issues associated with network implementation of our methods. Much of the work in aggregation of data by a network concerns network protocols for signal processing [13], managing constraints on bandwidth and energy [4], and dealing with errors or node failures [20]. This introductory paper does not treat these important issues. We also assume noise-free sensor readings. This paper assumes an idealized setting to develop and highlight the formal tools. 


\section{B. Outline}

The main results of this note consist of: (1) a theorem on target enumeration for continuum 'sensor fields' based on a topological integration theory; (2) bounds on the integrals for planar domains with holes; (3) a refinement theorem applicable to network discretizations; (4) a duality theorem for planar networks which provides a fast, distributed algorithm for ad hoc networks; (5) methods for computing expected target counts in the case of incomplete information; and (6) an outline of applications to time-dependent systems such as mobile robot sensing modalities. This note tersely summarizes ideas from [1], [2] in the restricted context of a planar network.

Our results follow from the classical and elegant theory of integration with respect to Euler characteristic [19], [16]. After surveying a simplified version of these methods in $\S \mathrm{II}$, we prove the fundamental enumeration theorem in $\S$ III. To solve the problem of sparse network discretization, we provide bounds in $\S \mathrm{V}$ on integrals over planar domains with a hole. This yields a simple refinement theorem in $\S \mathrm{VI}$, and extensions in $\S$ VII. We prove a duality result for planar networks in $\S$ VIII that leads to fast numerical implementation, outlined in $\S \mathrm{XI}$.

\section{TOPOLOGICAL INTEGRATION}

We present a simple, self-contained introduction to a topological integration theory. For simplicity, we work in the simplicial category. Let $X$ denote a simplicial complex: a topological space built from a collection of closed simplices glued together along faces (see, e.g., [9] for elementary definitions).

Definition 1: The EULER CHARACTERISTIC of a compact simplicial complex $X$ has two equivalent definitions:

1) combinatorial:

$$
\chi(X)=\sum_{k=0}^{\infty}(-1)^{k} \#\{k \text {-simplices in } X\} .
$$

2) homological:

$$
\chi(X)=\sum_{k=0}^{\infty}(-1)^{k} \operatorname{dim}\left(H_{k}(X)\right) .
$$

Here, $H_{k}(X)$ denotes the $k^{t h}$ (simplicial) homology of $X$ (in $R$ coefficients), a vector space that measures the number of 'holes' in $X$ that a $k$-dimensional subcomplex can detect [9]. As homology depends only on the homotopy type of $X$, the Euler characteristic $\chi$ is a topological invariant of a space, independent of how it is triangulated into a simplicial complex.

Example 2: The following examples are illustrative:

1) Euler characteristic is a generalization of cardinality: for a discrete set $X, \chi(X)=|X|$.

2) If $X$ is a compact contractible set - if it can be deformed continuously within itself to a single point then $\chi(X)=1$.

3) For a finite graph $\Gamma$, the Euler characteristic is $\chi(\Gamma)=$ $\# V(\Gamma)-\# E(\Gamma)$.

4) For $X \subset \mathbb{R}^{2}$ a connected set with $N$ holes, $\chi(X)=$ $1-N$
The Euler characteristic satisfies an inclusion-exclusion principle (a consequence of the Mayer-Vietoris sequence on homology [9]): for $A$ and $B$ compact subcomplexes of $X$,

$$
\chi(A \cup B)=\chi(A)+\chi(B)-\chi(A \cap B) .
$$

This equation evokes the definition of a measure and allows one to interpret $\chi$ as a generalized signed measure (generalized, as it is only finitely additive). As many authors have observed [15], [16], [19], this measure behaves as any conventional measure when restricted to the appropriate classes of integrands and domains. In the setting of $\mathbb{Z}$-valued functions over simplicial complexes, this measure theory is completely tame.

Definition 3: Let $X$ denote a simplicial complex and $C F(X)$ the abelian group of functions from $X$ to $\mathbb{Z}$ with generators $\mathbb{1}_{\sigma}$, where $\sigma$ is a closed simplex of $X$. Given such a $\phi=\sum_{\alpha} c_{\alpha} \mathbb{1}_{\sigma_{\alpha}}$ in $C F(X)$, the INTEGRAL of $\phi$ with respect to Euler characteristic is defined to be

$$
\int_{X} \phi d \chi:=\sum_{\alpha} c_{\alpha}
$$

This integral is well-defined.

Lemma 4 ([19], [16]): The integral $\int_{X} \phi d \chi$ depends only on the function $\phi$ and not on its decomposition. Specifically, if $\phi=\sum_{\alpha} c_{\alpha} \mathbb{1}_{U_{\alpha}}$, where $U_{\alpha}$ is a subcomplex of $X$, then

$$
\int_{X} \phi d \chi=\sum_{\alpha} c_{\alpha} \chi\left(U_{\alpha}\right) .
$$

Proof: Given two subcomplexes $A$ and $B$, the relation $\mathbb{1}_{A \cup B}=\mathbb{1}_{A}+\mathbb{1}_{B}-\mathbb{1}_{A \cap B}$ is mirrored by Equation (3). It follows that $\int_{X} \mathbb{1}_{U_{\alpha}} d \chi=\chi\left(U_{\alpha}\right)$. By definition, $\int_{X} \cdot d \chi$ is a homomorphism from $C F(X)$ to $\mathbb{Z}$; the lemma follows.

Remark 5: It is by no means necessary to restrict to simplicial complexes. For a large class of topological spaces without an explicit cell structure, $\chi$ is well-defined using Eqn. (2) with singular (or, better still, Borel-Moore) homology. Likewise, the class of integrable functions $C F(X)$ above generalizes to the sheaf of CONSTRUCTIBLE functions on $X$ [16]. This level of generality is not required for this paper.

\section{ENUMERATION VIA INTEGRATION}

We turn now to target-counting problems. The following mathematical formulation leads naturally to the integration theory of the previous section.

Consider a setting where the sensors are parameterized by a (reasonably nice) topological space $X$. One imagines a 'continuum field' setting in which a counting sensor resides at every point of $X$. It is helpful to keep in mind two cases: (1) $X=\mathbb{R}^{2}$ and is 'filled' with sensors; (2) $X$ is a simplicial complex, where the counting sensors at the vertices of $X$ 'pass' counting data to all other simplices of $X$. Assume a finite set of stationary targets are present and detectable by the sensor field. We do not specify detection ranges, etc., in terms of geometric constraints, but rather in terms of sets. For each target $\alpha$, define its TARGET SUPPORT, $U_{\alpha} \subset X$, to be the subset of those sensors to which the target is 'visible' (rather, 
sensed: the actual sensor modality is irrelevant). The sensor field on $X$ returns a counting function $h: X \rightarrow \mathbb{N}$, where

$$
h(x)=\#\left\{\alpha: x \in U_{\alpha}\right\} .
$$

Assuming knowledge of the target supports' topology, one has a simple means of enumerating the targets without localization or identification.

Theorem 6: If each target has a compact contractible support $\mathcal{U}_{\alpha} \subset X$, then the integral of the impact function $h(x)=$ $\#\left\{\alpha: x \in U_{\alpha}\right\}$ with respect to $d \chi$ is the target count:

$$
\# \alpha=\int_{X} h d \chi \text {. }
$$

Proof: By definition, $h=\sum_{\alpha} \mathbb{1}_{U_{\alpha}}$. As $U_{\alpha}$ is compact and contractible, $\chi\left(U_{\alpha}\right)=1$ and $\int_{X}^{\alpha} h d \chi=\sum_{\alpha} 1=\# \alpha$.

The remarkable aspect of this result is that there are no constraints on the target supports other than the topological: each target has support with $\chi=1$. In particular, targets can have different 'impact' on the sensor field, and there is no need for convexity or fixed-radius assumptions.

Theorem 6 does, however, assume that there is a welldefined counting function $h$ over all of $X-e . g$., given by a sensor at every point in the space $X$. In a less idealized setting, one has a finite number of nodes which, under the best circumstances, triangulates a region of the plane. The integrand 'counting function' $h$ is known only on the vertex set of this triangulation $\mathcal{T}$. The target supports $U_{\alpha} \subset \mathbb{R}^{2}$ need not be well-placed with respect to $\mathcal{T}$ at all.

We resolve this discretization problem by extending the integration theory to $\mathbb{R}$-valued integrands. By taking the usual step-function upper semi-continuous approximation to a limit, one can define $\int h d \chi$ for real-valued functions $h: X \rightarrow$ $[0, \infty)$ which are reasonably behaved (e.g., which have a finite number of critical points). This extension of the theory is not without complications (e.g., the integration operator is no longer linear), but it allows one to import perspectives from numerical analysis. In particular, given a sampling of an integrand $h$ over a discrete set, the integral of the piecewiselinear (PL) interpolation of $h$, denoted $h_{P L}$, should be a good approximation if the sampling is of sufficient fidelity. This holds for integration with respect to $d \chi$.

Theorem 7: Fix a collection $\left\{U_{\alpha}\right\}$ of compact target supports in $\mathbb{R}^{n}$ in general position. For a triangulation $\mathcal{T}$ of $\mathbb{R}^{n}$, let $h_{P L}$ denote the piecewise-linear extension of the restriction of $h=\sum_{\alpha} \mathbb{1}_{U_{\alpha}}$ to the vertices of $\mathcal{T}$. Then, for $\mathcal{T}$ sufficiently fine and regular,

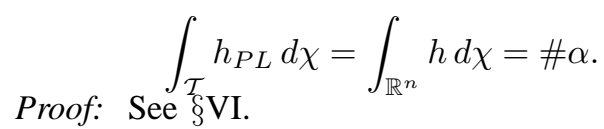

\section{Computation}

Theorems 6 and 7 are useless without effective means of computing integrals with respect to $d \chi$. Fortunately, there are several means of doing so.

Theorem 8: Given a compactly supported impact function $h: X \rightarrow \mathbb{N}$, the integral of $h$ with respect to $d \chi$ may be computed as:

$$
\begin{aligned}
\int_{X} h d \chi & =\sum_{s=0}^{\infty} \chi(\{h>s\}) \\
& =\int_{s=0}^{\infty} \chi(\{h \geq s\}) d s \\
& =\sum_{p \in \mathcal{C}(h)}(-1)^{n-\mu(p)} h(p)
\end{aligned}
$$

Eqns. (8) and (9) apply to $\mathbb{N}$-valued and $[0, \infty)$-valued impact functions respectively, and the notation $\{h>s\}$ represents the set $h^{-1}((s, \infty))$. Eqn. (10) applies to a $[0, \infty)$-valued Morse function on an $n$-dimensional manifold, where $\mathcal{C}(h)$ is the set of critical points of $h$, and $\mu(p)$ is its Morse index of $p \in \mathcal{C}(h)$ [14].

Proof: Eqns. (8) and (9) are elementary and follow directly from the definitions. For Eqn. (10), one has $h$ Morse. Thus, the Euler characteristic of upper excursion sets is piecewise-constant, changing only at critical values. For $p \in$ $\mathcal{C}(h), s=h(p)$, and $\epsilon \ll 1$, elementary Morse theory [14] says that $\{h \geq s+\epsilon\}$ differs from $\{h \geq s-\epsilon\}$ by the addition of a product of discs $D^{\mu(p)} \times D^{n-\mu(p)}$ glued along $D^{\mu(p)} \times \partial D^{n-\mu(p)}$. The change in Euler characteristic resulting from this handle addition is $(-1)^{n-\mu(p)}$. This, applied to Eqn. (8) yields Eqn. (10).

This theorem means, roughly speaking, that one can trade between Euler characteristic counts, integrals with respect to Lebesgue measure, and Morse theory at will.

Fig. 1(a) gives an example of a collection of target supports $\left\{U_{\alpha}\right\}$ with height function, which is sampled on a uniform hexagonal grid in (b). The upper excursion sets of $h$ are easily computed and the integral with respect to Euler characteristic is thus:

$$
\# \alpha=\int h d \chi=\overbrace{1}^{s=2}+\overbrace{3}^{s=1}+\overbrace{0}^{s=0}=4 .
$$

Smoothing $h$ to a function $\tilde{h}$ with nondegenerate critical points yields three maxima and three saddles, with minima (at height zero), see Fig. 1(c). Formula (10) implies

$$
\int h d \chi=\overbrace{(3+2+2)}^{\mu=2}-\overbrace{(1+1+1)}^{\mu=1}+\overbrace{0}^{\mu=0}=4 .
$$

Taking the PL extension $h_{P L}$ yields upper excursion sets as illustrated in Fig. 1(d): Eqn. (9) yields a computation similar to that of (11).

\section{HOLES IN THE NETWORK}

It is common in sensor networks to encounter 'holes' within the network, through incomplete coverage or node failures. In this case, one wants to estimate the number of targets relative to the missing information. This translates to the following relative problem: if one knows $h: X \rightarrow \mathbb{N}$ only on some subset $A \subset X$, how well can one estimate $\int_{X} h d \chi$ from the restriction $h \mid A$ ? We give bounds for the planar case. 


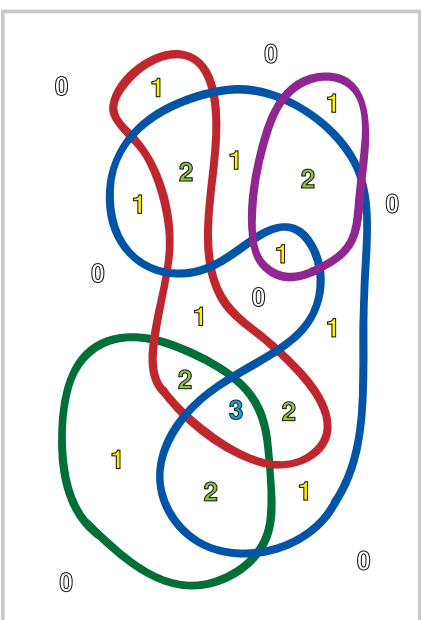

(a)

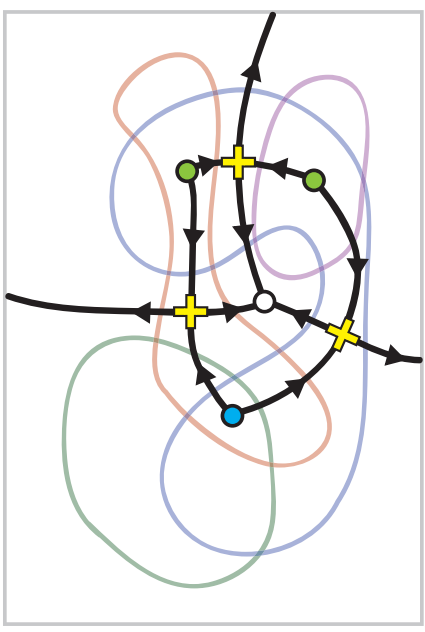

(c)

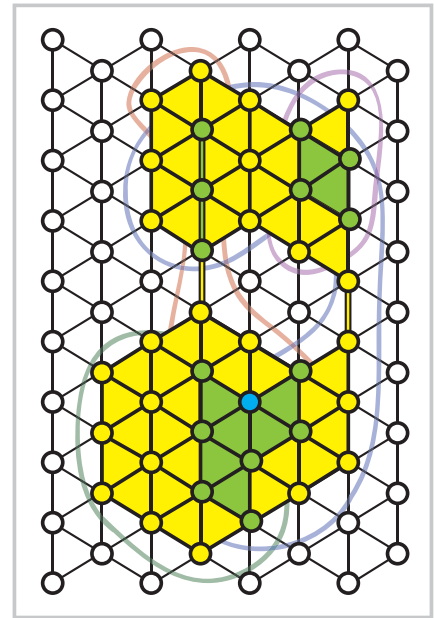

(b)

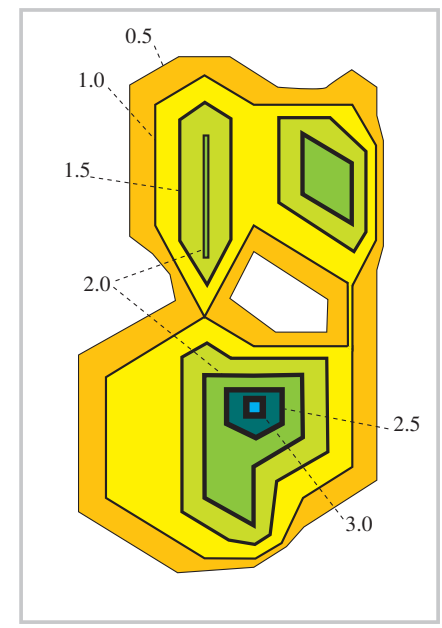

(d)
Fig. 1. The height function of a collection of target supports (a) is sampled on a regular triangulation (b). This can be smoothed to a Morse function with maxima/saddles/minima (c) or extended over the triangulation via PL interpolation (d).

Theorem 9: Assume $h: \mathbb{R}^{2} \rightarrow \mathbb{N}$ is the sum of indicator functions over a collection of compact contractible sets in $\mathbb{R}^{2}$, none of which is contained entirely within $D$, a fixed open contractible disc. Then

$$
\int_{\mathbb{R}^{2}} \hat{h} d \chi \leq \int_{\mathbb{R}^{2}} h d \chi \leq \int_{\mathbb{R}^{2}} \check{h} d \chi
$$

where

$$
\begin{aligned}
& \hat{h}(y)=\left\{\begin{array}{cl}
\max _{\partial D} h & : y \in \bar{D} \\
h & : \text { else }
\end{array}\right. \\
& \check{h}(y)=\left\{\begin{array}{cl}
\min _{\partial D} h & : y \in D \\
h & : \text { else }
\end{array}\right.
\end{aligned}
$$

Proof: Via additivity of $\chi$ over domains, Eqn. (13) follows from the corresponding inequalities over the compact domain $\bar{D}$. Explicitly, if $\bar{h}=h$ on $\mathbb{R}^{2}-\bar{D}$, then

$$
\int_{\mathbb{R}^{2}} \bar{h} d \chi=\int_{\mathbb{R}^{2}-D} h d \chi-\int_{\partial D} h d \chi+\int_{\bar{D}} \bar{h} d \chi .
$$

Denote by $\mathcal{V}=\left\{V_{\beta}\right\}$ the collection of nonempty connected components of intersections of all target supports $U_{\alpha}$ with $\bar{D}$. Since we work in $\mathbb{R}^{2}$, each $V_{\beta}$ is a compact contractible set which intersects $\partial D$. By Theorem $6, \int_{\bar{D}} h d \chi$ equals the number of components $|\mathcal{V}|$. There are at least $\max _{\partial D} h$ such pieces; hence

$$
\int_{\bar{D}} \hat{h} d \chi \leq \int_{\bar{D}} h d \chi
$$

Consider $\min _{\partial D} h$ and remove from the collection $\mathcal{V}$ this number of elements, including all such $V_{\beta}$ equal to $\bar{D}$ (which is possible since we remove $\min _{\partial D} h$ such elements). Each remaining $V_{\beta} \in \mathcal{V}$ is not equal to $\bar{D}$ and thus intersects $\partial D$ in a set with strictly positive Euler characteristic. Thus,

$$
\int_{\bar{D}} h d \chi \leq \int_{\bar{D}} \check{h} d \chi
$$
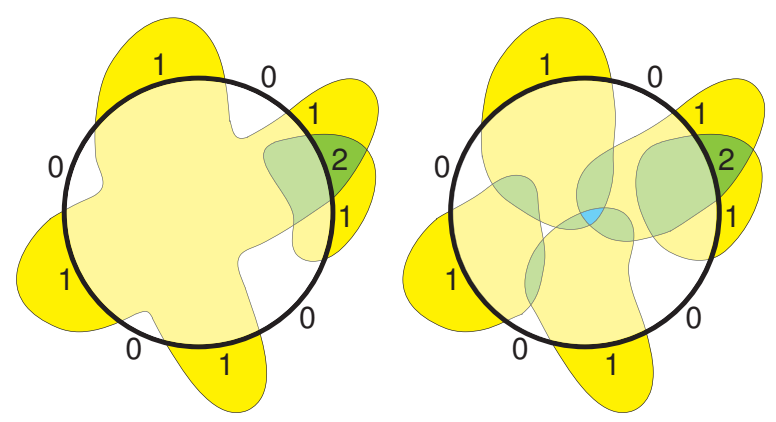

Fig. 2. An example for which the upper and lower bounds of Eqn. (13) are sharp.

Example 10: Consider the example illustrated in Fig. 2. The upper and lower estimates for the number of targets are 2 and 4 respectively. For this example, the estimates are sharp in that one can have collections of target supports over compact contractible sets which agree with $h$ outside of $\bar{D}$ and realize the bounds.

Remark 11: The lower bound $\hat{h}$ can fail in several ways. For example, a target support can intersect $D$ in multiple components, causing $\hat{h}$ to not have a decomposition as a sum of characteristic functions over contractible sets (but rather with annuli). One can even find examples for which each target support intersects $\bar{D}$ in a contractible set but for which $\int \hat{h} d \chi$ is negative. The fact that the lower bound $\int \hat{h}$ can be so defective follows from the difficulty associated with annuli in the plane - these are 'large sets of measure zero' in $d \chi$.

\section{THE REFINEMENT THEOREM}

The bounds of $\S \mathrm{V}$ allow one to conclude when a hole is 'inessential' and no ambiguity about the integral exists.

Corollary 12: Under the hypotheses of Theorem 9, the upper and lower bounds are equal when there is a unique connected local maximum of $h$ on $\partial D$.

Proof: In the case where $h$ is constant on $\partial D, \breve{h}=\hat{h}$ and the result is trivial. Otherwise, both $\breve{h}$ and $\hat{h}$ have connected 
(and thus contractible) upper excursion sets. Applying Eqn. (8) yields

$$
\begin{aligned}
\int_{\mathbb{R}^{2}} \check{h} d \chi-\int_{\mathbb{R}^{2}} \hat{h} d \chi & =\sum_{s} \chi(\{\check{h} \geq s\})-\chi(\{\hat{h} \geq s\}) \\
& =\sum_{s} 1-1=0 .
\end{aligned}
$$

This permits an easy proof of Theorem 7 that, in accordance with one's intuition about integration, refinement of the network leads to convergence of the integrals.

Proof: (of Theorem 7) Since integration is local, we may compare $\int h d \chi$ and $\int h_{P L} d \chi$ over a single closed 2simplex of the triangulation $\mathcal{T}$ : if these are always equal, then the theorem follows. One observes that for the $U_{\alpha}$ in general position and $\mathcal{T}$ sufficiently fine and regular, the unique local maxima of $h$ and $h_{P L}$ on the boundary of any 2-simplex of $\mathcal{T}$ are equal and both level sets are connected. Corollary 12 completes the proof.

It is easy to extend this proof to $\mathbb{R}^{n}$ by proving the appropriate extension of Corollary 12.

\section{HARMONIC EXTENSION AND EXPECTED TARGET COUNTS}

We continue the results of the previous section, considering the case of a planar domain with a contractible hole on which the integrand is unknown. As shown, upper and lower bounds are realized by extending the integrand across the hole via minimal and maximal values on the boundary of the hole. Inspired by the result that the PL-extension of a discretely sampled integrand yields correct integrals with respect to Euler characteristic, we consider extensions over holes via continuous functions.

The following result says that there is a principled interpolant between the upper and lower extensions. Roughly speaking, an extension to a harmonic function (discrete or continuous, solved over the hole with Dirichlet boundary conditions) provides an approximate integrand whose integral lies between the bounds given by upper and lower convex extensions. There is nothing magical about harmonic functions: any form of weighted averaging will lead to an extension which respects the bounds. A specific criterion follows.

Theorem 13: Given $h: \mathbb{R}^{2}-D \rightarrow \mathbb{N}$ satisfying the assumptions of Theorem 9, let $\bar{h}$ be any extension of $h$ which has no strict local maxima or minima on $D$. Then

$$
\int_{\mathbb{R}^{2}} \hat{h} d \chi \leq \int_{\mathbb{R}^{2}} \bar{h} d \chi \leq \int_{\mathbb{R}^{2}} \check{h} d \chi
$$

Proof: Consider an open neighborhood of $\bar{D}$ in $\mathbb{R}^{2}$ and modify $\bar{h}$ so that it preserves critical values, is Morse, and falls off to zero quickly outside of $D$. This perturbed function, denoted $\tilde{h}$, has isolated maxima on $\partial D$, isolated saddles in the interior of $D$ (since there are no local extrema in $D$ by hypothesis) and no other critical points outside of $D$. Since $\tilde{h}$ is a small perturbation of $\bar{h}$, the integral of $\tilde{h}$ with respect to $d \chi$ is equal to $\int \bar{D} \bar{h} d \chi$. Via the Morse-theoretic formula of Eqn. (10),

$$
\int \tilde{h} d \chi=\sum_{p \in \mathcal{C}(\tilde{h})}(-1)^{2-\mu(p)} \tilde{h}(p) .
$$

The integral thus equals the sum of $h$ over the maxima on $\partial D$ minus the sum of $\bar{h}$ over the saddle points in the interior of $D$, since saddles have Morse index $\mu=1$.

Denote by $\left\{p_{i}\right\}_{1}^{M}$ the maxima of $\tilde{h}$, ordered by their (increasing) $\tilde{h}$ values. Denote by $\left\{q_{i}\right\}_{1}^{N}$ the saddles of $\tilde{h}$, ordered by their (increasing) $\tilde{h}$ values. By the Poincaré index theorem,

$$
1=\chi(\bar{D})=\# \operatorname{maxima}(\tilde{h})-\# \operatorname{saddles}(\tilde{h}),
$$

hence, $N=M-1$. Note that, since there are no local minima, $\tilde{h}\left(q_{i}\right)<\tilde{h}\left(p_{i}\right)$ for all $i=1 \ldots M-1$. Thus,

$$
\begin{aligned}
\int_{\bar{D}} \bar{h} d \chi & =\int_{\bar{D}} \tilde{h} d \chi \\
& =\tilde{h}\left(p_{M}\right)+\sum_{i=1}^{M-1} \tilde{h}\left(p_{i}\right)-\tilde{h}\left(q_{i}\right) \\
& \geq \tilde{h}\left(p_{M}\right)=\max _{\partial D} h=\int_{\bar{D}} \hat{h} d \chi .
\end{aligned}
$$

For the other bound,

$$
\begin{aligned}
\int_{\bar{D}} \bar{h} d \chi & =\tilde{h}\left(p_{M}\right)+\sum_{i=1}^{M-1} \tilde{h}\left(p_{i}\right)-\tilde{h}\left(q_{i}\right) \\
& \leq \sum_{i=1}^{M} \tilde{h}\left(p_{i}\right)=\int_{\bar{D}} \check{h} d \chi .
\end{aligned}
$$

A harmonic or harmonic-like function $\tilde{h}$ will often lead to an integral with non-integer value. Such an integral is best interpreted as an expected target count.

Example 14: Consider a hole $D$ and a function $h$ which is known only on $\partial D$ and which has two maxima with value 1 and two minima with value 0 . Without knowing more about the possible size and shape of the target supports which make up $h$, it is not clear whether this is more likely to come from one target support (which crosses the hole) or from two separate target supports. Computing a harmonic extension of this $h$ over the interior of $D$ yields a function $\tilde{h}$ with one saddle-type critical point in $D$. The value of the saddle is $c$ and satisfies $0<c<1$, depending on the geometry of $h$ on $\partial D$. This yields $\int \bar{h} d \chi=2-c$, reflecting the uncertainty of either one or two targets. In the perfectly symmetric case of Fig. 3[left], $c=\frac{1}{2}$ and the expected target count is, naturally, $\frac{3}{2}$. In Fig. 3 [right], the harmonic extension has $c<\frac{1}{2}$, meaning that it is more likely that there are two target supports.

In the network setting, holes often arise due to node failure or lack of sufficient node density. In these scenarios, one may reasonably employ any weighted local averaging scheme across dead nodes to recover a function which will respect the bounds of Theorem 9. Different weighting schemes may 

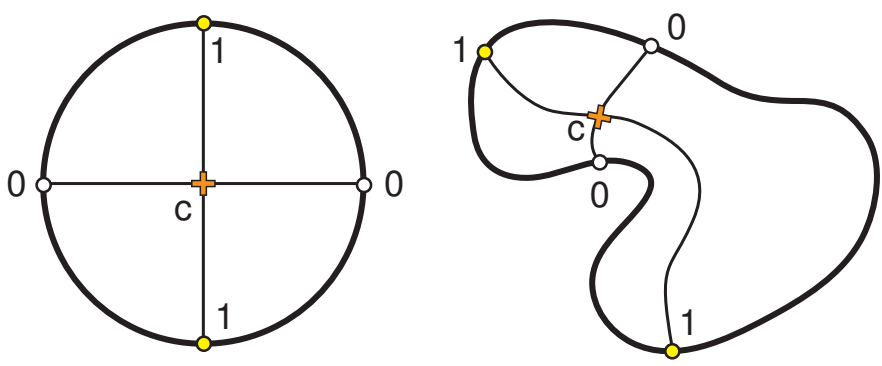

Fig. 3. An integrand with a hole has two minima at height 0 and two maxima at height +1 . Filling in by a harmonic function $\tilde{h}$ has an interior saddle at height $0<c<1$, depending on the geometry of $h$ on $\partial D$ : [left] $c=\frac{1}{2}$; [right] $c<\frac{1}{2}$.

be more appropriate for different systems. For example, node readings can be assigned a "confidence" measure, which, when used as a weighting for the averaging over the dead zone, returns an expected value of the integral which reflects the fidelity of the data.

\section{HOLES VIA DUALITY}

We augment Theorem 8 with a specialized formula for the plane which aids greatly with implementation. The strategy of this formula is to exploit the duality between holes and connected components of the complement. This duality has a formal expression in terms of algebraic topology.

Theorem 15: For $h: \mathbb{R}^{2} \rightarrow \mathbb{N}$

$$
\int_{\mathbb{R}^{2}} h d \chi=\sum_{s=0}^{\infty}\left(\beta_{0}\{h>s\}-\beta_{0}\{h \leq s\}+1\right),
$$

where $\beta_{0}$ is the number of connected components of the set.

Proof: Let $A$ be a compact nonempty subset of $\mathbb{R}^{2}$. Since $A \subset \mathbb{R}^{2}, H_{s}(A)=0$ for all $s \geq 2$. Thus, via Eqn. 2, it suffices to compute

$$
\chi(A)=\operatorname{dim} H_{0}(A)-\operatorname{dim} H_{1}(A) .
$$

Note: $\operatorname{dim} H_{0}$ equals the number of connected components of $A$. The quantity $\operatorname{dim} H_{1}(A)$, the number of holes in $A$, is, by Alexander duality [9], equal to $\operatorname{dim} H_{0}\left(\mathbb{R}^{2}-A\right)-$ 1 , the number of (bounded) connected components of the complement. The proof is completed by Eqn. (8), substituting in $A=\{h>s\}$ and $\mathbb{R}^{2}-A=\{h \leq s\}$.

Example 16: The duality formula (15) applied to the integrand of Fig. 1 yields

$$
\int_{\mathbb{R}^{2}} h d \chi=\overbrace{1-2+1}^{s=0}+\overbrace{3-1+1}^{s=1}+\overbrace{1-1+1}^{s=2}=4 .
$$

The formula in Theorem 15 is extremely applicable. We note that the determination of the number of connected components of the upper and lower excursion sets is a simple clustering problem, computable in logspace with respect to the number of network nodes.

\section{AD HOC NETWORKS}

We note that the strategy of converting the sampling of the true impact function $h$ over $\mathcal{N}$ to a PL interpolation $\bar{h}$ does not necessarily require knowing the coordinates of the nodes. Indeed, the evaluation of $\int_{Y} \cdot d \chi$ is conspicuous in its freedom from coordinate geometry: it is a topological integral. If one is given a triangulation, the extension of the counting function $h$ on vertices over the domain is automatic. However, if no geometry associated to $\mathcal{N}$ is known, it may not be possible to determine a canonical extension $h_{P L}$ over the domain. Such a situation is not uncommon in sensor networks based on ad hoc wireless communications, an increasingly common protocol for distributed sensor networks and robotics.

Assume that one is given a network in the form of an abstract graph $\mathcal{G}=(\mathcal{N}, \mathcal{E})$. By "abstract" we mean that the projection of the $1-d$ cell complex $\mathcal{G}$ to the workspace is unknown. Edges should possess some proximity data. For example, one could assume that $\mathcal{G}$ is a UNIT DISC GRAPH, in which edges exist between nodes if and only if they are within unit distance in the workspace. A more realistic model is the QUASI unit disc graph, in which edges definitely exist below a certain distance, definitely do not exist above a certain distance, and may exist (say, according to some probability distribution) for nodes within a critical interval of distance. At any rate, the duality results of $\oint$ VIII allow us to compute integrals based on ad hoc networks.

Corollary 17: Assume an integrand $h: \mathbb{R}^{2} \rightarrow \mathbb{N}$, and let $\mathcal{G}$ be a network graph with nodes $\mathcal{N} \subset \mathbb{R}^{2}$, where the only thing known is the restriction of $h$ to $\mathcal{N}$ (in particular, the coordinates of $\mathcal{N}$ in $\mathbb{R}^{2}$ are unknown). If the network $\mathcal{G}$ correctly samples the connectivity of the upper and lower excursion sets of $h$, then Eqn. (15) returns the exact number of targets.

An example appears in Fig. 4. Note that in this example, the topology of the excursion sets of $h$ are not sampled correctly: sparsity leads to holes in the network. Nevertheless, since the connectivity of the upper and lower excursion sets is sampled faithfully, the integral is correct. Although the example drawn is a unit disc graph, this is by no means necessary for the result.

\section{Mobile Agents}

The setting of this work has assumed stationary targets with fixed target supports, being sensed by a fixed network of stationary counting sensors. It is desirable to violate both assumptions, especially in the robotics context. We indicate how the results of this note are applicable to both settings in a sequence of remarks.

Remark 18: Consider the following scenario: a collection of fixed target supports $\left\{U_{\alpha}\right\}$ lie in the plane. One or more mobile robots $R_{i}$ can maneuver in the plane along chosen paths $x_{i}(t)$, returning sensed counting functions $h_{i}(t)=$ $\#\left\{\alpha: x_{i}(t) \in U_{\alpha}\right\}$. How should the paths $x_{i}$ be chosen so as to effectively determine the correct target count? If target supports are extremely convoluted, no guarantees are possible: therefore, assume that some additional structure is known (e.g., 


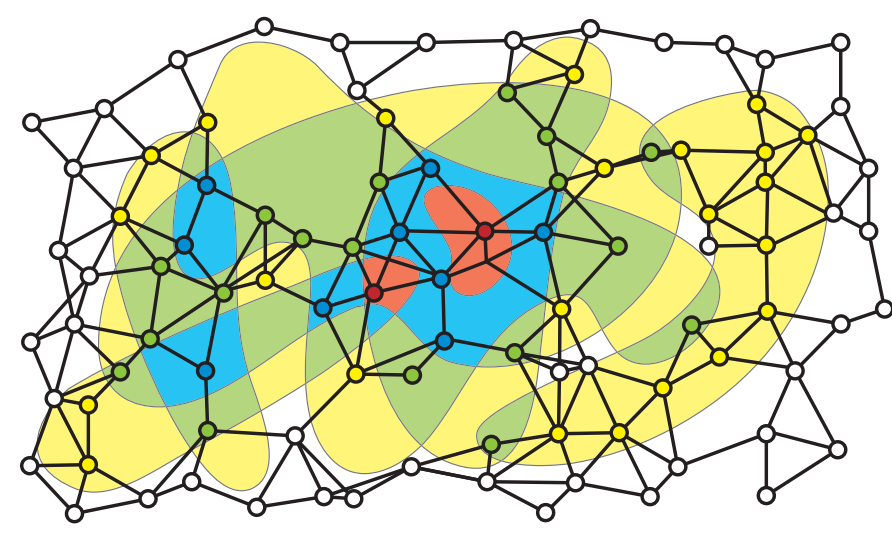

Fig. 4. A sparse sampling over an ad hoc network retains enough connectivity data to evaluate the integral exactly.

an injectivity radius) giving a lower bound on how "thin" the target supports may be.

Assume that the robots initially explore the planar domain along a rectilinear graph $\Gamma$ that tiles the domain into rectangles. If desired, one can make these rectangles have either width or height in order to guarantee that all the $U_{\alpha}$ intersect $\Gamma$. Consider the sensor function $h: \Gamma \rightarrow \mathbb{N}$. The integral $\int_{\Gamma} h d \chi$ is likely to give the wrong answer, even (especially!) for a dense $\Gamma$. Two means of getting a decent approximation are (1) use the duality formula of Eqn. (15); or (2) perform a harmonic extension over the holes of $\Gamma$ as per $\S$ VII.

However, neither is guaranteed to give a good a priori approximation to the target count. How can one tell if $\Gamma$ should be filled in more? The simplest criterion follows from Corollary 12. Consider a basic cycle $\Gamma^{\prime} \subset \Gamma$ in the tiling induced by $\Gamma$. If there is a single connected local maximum on $\Gamma^{\prime}$, then (assuming that no small $U_{\alpha}$ lies entirely within the hole) the harmonic extension over $\Gamma^{\prime}$ gives an accurate contribution to the integral.

If, on the other hand, there are multiple maximal sets on $\Gamma^{\prime}$, then one must refine $\Gamma$ into smaller cycles for which the criterion holds. The obvious approach is to guide the mobile sensors so as to try and connect disjoint maxima and/or disjoint minima. Fig. 5 gives the sense of the technique. We leave for future work detailing a complete algorithm and its analysis: the crucial observation is that Corollary 12 provides a stopping criterion.

Remark 19: One can imagine a much more complicated scenario. Consider the case where the target supports also vary (continuously) as a function of time: $U_{\alpha}(t) \subset \mathbb{R}^{2}$. However, the supports are unknown to the robots $R_{i}$, which can measure only a sampled count $h_{i}(t)$.

The problem is clearly unsolvable if there is a single, slow robot: such a sensor may never detect any (evasive) targets at all. On the other hand, if one assumes a dense network of sensors, the problem is trivial: at any fixed time, take a triangulation of the domain based on the robot positions, and compute the integral of the sensor function as per Theorem 7.

Where the problem is critically difficult is when the swarm

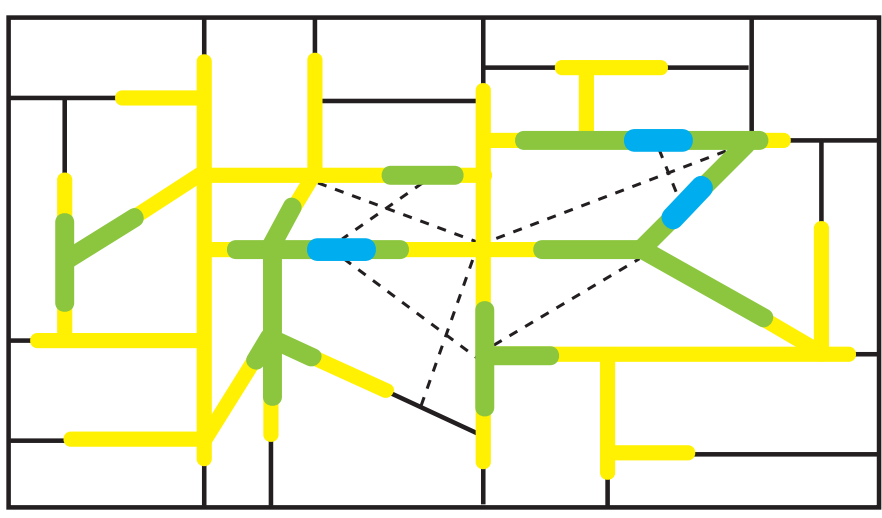

Fig. 5. Mobile agents determine target counts over a graph $\Gamma$. Holes with multiple maxima require further refinement (dashed lines).

of sensors is not dense enough to cover the plane, but does form a connected network with holes. These holes will change temporally, emerging, bifurcating, disappearing: all the while, mobile targets can slip in and out.

In this dynamic setting, the work in $\S \mathrm{V}-\mathrm{VII}$ suggests a natural strategy of computing an expected value of the integral as a function of time and keeping a running average of these approximants. More sophisticated tracking of targets within holes can be accomplished by examining localized temporal discontinuities of these integrals. This is the subject of a separate report.

Our discussion of mobile agents is necessarily brief: there are many more results possible about counting mobile targets without the need of clocks at all [1]. We leave these and implementation issues for a more detailed future treatment.

\section{NUMERICAL ISSUES}

Space constraints forbid a comprehensive treatment of the topic of numerical integration with respect to Euler characteristic, a topic which seems to have been explored only in [12], and here from an integral-geometry perspective: there is much to be done. We present a few significant remarks, and leave the details for an archival work.

Remark 20: Implementation. We have implemented the integration formula of $\S$ VIII, Eqn. (15), for ad hoc planar networks based on a random unit disc graph: see Fig. 6. The code (written in Java and publicly available at [hidden for review]) allows the user to specify target support by drawing with the mouse. By using the obvious clustering algorithm, the code returns the quantity specified in Eqn. (15) in negligible time ( $\sim 1$ s for a network of $\sim 100,000$ nodes $)$.

Remark 21: Numerical errors. Of course, the guarantee that Eqn. (15) computes the correct value of the integral depends on having sampled the connectivity of the upper and lower excursion sets correctly. No a priori knowledge of this can be assured without knowing more about the network or the target support. Unfortunately, the duality formula computes a $\mathbb{Z}$-valued sum, any error in the computation is quantized. From the point of view of numerical errors, it is preferable to 
work with the 'expected' $\mathbb{R}$-valued integrals as in Eqn. (10) and $\S$ VII.

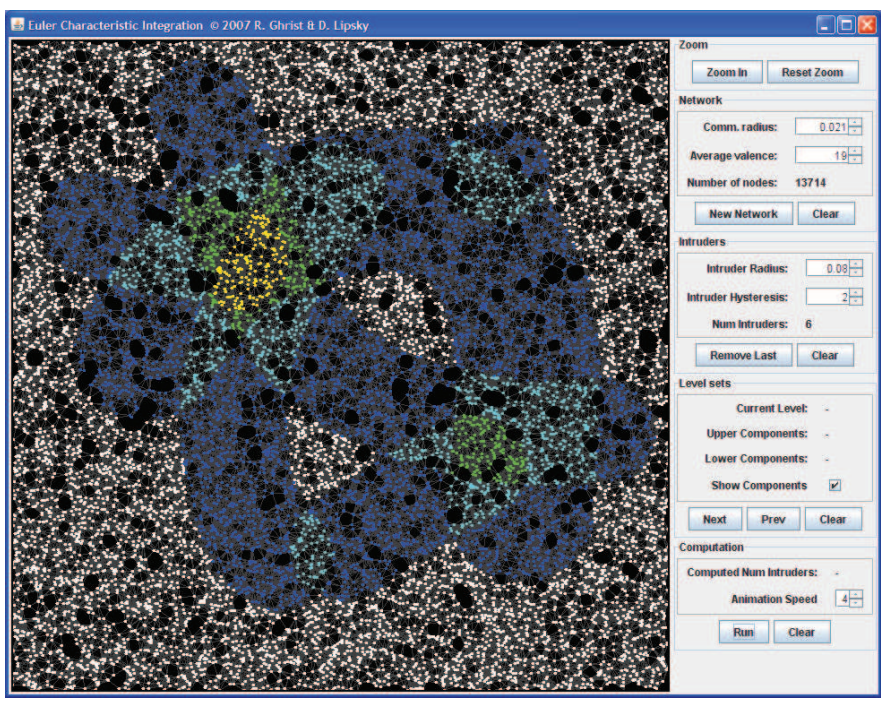

Fig. 6. Screenshot of a Java applet implementation [written by D. Lipsky], cf. Remark 20.

Remark 22: Distributed computation. Since our methods are based on an integration theory, the enumeration of targets detailed in this paper is a local computation. For $A, B$ compact,

$$
\int_{A \cup B} h d \chi=\int_{A} h d \chi+\int_{B} h d \chi-\int_{A \cap B} h d \chi .
$$

Thus, enumeration can be performed in a distributed manner easily. This is particularly easy when the network is a lattice, as one can employ standard distributed protocols for localization and merging of target counts.

\section{CONCLUDING REMARKS}

The core message of this paper is that thinking of target enumeration in terms of a topological integration theory is much better than a raw combinatorial approach. One can import intuition, techniques, and perspectives from numerical analysis, algebraic topology, differential topology, and combinatorics at will.

This short paper has left many natural questions unanswered. We give a brief list of questions and remarks, to be expanded on in future papers.

Remark 23: Do these results extend to higher dimensions? Yes, and to reasonable topological spaces as well. The results on bounds for holes and the duality formula, unfortunately, do not generalize, being dependent on planar topology.

Remark 24: What about noise? This integration theory is robust to dead sensors: an empty node creates a 'hole' which the techniques of this paper resolve. However, as this integration theory counts the number and heights of critical points, it is very sensitive to integer-valued noise. A smoothing filter is required to preprocess noisy data in order to obtain accurate results.
Remark 25: How do you know if you've sampled the domain finely enough? As in the case of trying to approximate the Riemann integral of an unknown function from a finite point sample, one does not know without more data.

Remark 26: What about sensors which do not count but rather measure $[0, \infty)$-valued intensity? This integration theory is not immediately applicable, since the operator $\int \cdot d \chi$ is not linear on continuous integrands. However, one can obtain lower bounds using methods akin to Lusternik-Schnirelmann category [3].

We hope the reader finds that the increase in formalism for this integration theory more than pays for itself in terms of potential applications.

\section{ACKNOWLEDGEMENTS}

This work is funded by DARPA \# HR0011-05-1-0008.

\section{REFERENCES}

[1] Y. Baryshnikov and R. Ghrist, "Target enumeration via Euler characteristic integrals I: sensor fields," to appear, SIAM J. Appl. Math.

[2] Y. Baryshnikov and R. Ghrist, "Target enumeration via Euler characteristic integrals II: sensor networks," in preparation.

[3] Y. Baryshnikov and R. Ghrist, "Unimodal category and the topological decomposition of distributions," in preparation.

[4] A. Boulis, S. Ganeriwal, and M. Srivastava, "Aggregation in sensor networks: an energy - accuracy tradeoff," J. Ad-hoc Networks, 1, 2003, 317-331.

[5] D. Estrin, D. Culler, K. Pister, and G. Sukhatme, "Connecting the Physical World with Pervasive Networks," IEEE Pervasive Computing $1: 1,2002,59-69$.

[6] Q. Feng, F. Zhao, And L. Guibas, "Lightweight Sensing and Communication Protocols for Target Enumeration and Aggregation" in proceedings MobiHoc, 2003.

[7] H. Groemer, "Minkowski addition and mixed volumes," Geom. Dedicata 6, 1977, 141-163.

[8] L. Guibas. "Sensing, Tracking and Reasoning with Relations," IEEE Signal Processing Magazine, 19(2), Mar 2002,

[9] A. Hatcher, Algebraic Topology, Cambridge University Press, 2002.

[10] T. He, P. Vicaire, T. Yan, L. Luo, L. Gu, G. Zhou, R. Stoleru, Q. Cao, J. Stankovic, T. Abdelzaher, "Achieving Real-Time Target Tracking Using Wireless Sensor Networks," in proceedings of IEEE Real Time Technology and Applications Symposium, 2006, 37-48.

[11] B. Jung and G. Sukhatme, "A Region-Based Approach for Cooperative Multi-Target Tracking in a Structured Environment," in proceedings of IEEE/RSJ Conference on Intelligent Robots and Systems, 2002.

[12] D. Klain, K. Rybnikov, K. Daniels, B. Jones, C. Neacsu, "Estimation of Euler Characteristic from Point Data," preprint 2006.

[13] D. Li, K. Wong, Y. Hu, and A. Sayeed, "Detection, classification, and tracking of targets," IEEE Signal Processing Magazine, 19(2), 2002, $17-30$.

[14] J. Milnor, Morse Theory, Princeton University Press, 1963.

[15] G.-C. Rota, "On the combinatorics of the Euler characteristic," Studies in Pure Mathematics, Academic Press, London, 1971, 221-233.

[16] P. Schapira, "Operations on constructible functions," J. Pure Appl. Algebra 72, 1991, 83-93.

[17] P. Schapira, "Tomography of constructible functions," in proceedings of 11th Intl. Symp. on Applied Algebra, Algebraic Algorithms and ErrorCorrecting Codes, 1995, 427-435.

[18] J. Singh, U. Madhow, R. Kumar, S. Suri, and R. Cagley, "Tracking multiple targets using binary proximity sensors." In Proceedings of the 6th international Conference on Information Processing in Sensor Networks, 2007, 529-538.

[19] O. Viro, "Some integral calculus based on Euler characteristic," Lecture Notes in Math., vol. 1346, Springer-Verlag, 1988, 127-138.

[20] J. Zhao, R. Govindan and D. Estrin, "Computing Aggregates for Monitoring Wireless Sensor Networks," in proceedings of IEEE Intl. Workshop on Sensor Network Protocols and Applications (SNPA), 2003. 